\begin{tabular}{|c|c|}
\hline Title & Finite Element Solution of Horizontally Polarized Shear W ave Scattering in an Elastic Plate \\
\hline Author(s) & Koshiba, M.; Hasegawa, K.; Suzuki, M. \\
\hline Citation & IEEE Transactions on UItrasonics, Ferroelectrics and Frequency Control, 34(4), 461-466 \\
\hline Issue Date & $1987-07$ \\
\hline Doc URL & http:/hdl. handle.net/2115/7369 \\
\hline Rights & $\begin{array}{l}\text { O1987 IEEE. Personal use of this material is permitted. However, permission to reprint/republish this material for } \\
\text { advertising or promotional purposes or for creating new collective works for resale or redistribution to servers or lists, } \\
\text { or to reuse any copyrighted component of this work in other works must be obtained from the IEEE. } \\
\text { IEEE, IEEE Transactions on Ultrasonics, Ferroelectrics and Frequency Control, 34(4), 1987, p461-466 }\end{array}$ \\
\hline Type & article \\
\hline File Information & ITUFFC34_4.pdf \\
\hline
\end{tabular}

Instructions for use 


\title{
Finite-Element Solution of Horizontally Polarized Shear Wave Scattering in an Elastic Plate
}

\author{
MASANORI KOSHIBA, SENIOR MEMBER, IEEE, KOJI HASEGAWA, AND \\ MICHIO SUZUKI, SENIOR MEMBER, IEEE
}

\begin{abstract}
A method of the solution of scattering of horizontally polarized shear waves in an elastic plate is described. The approach is a combination of the finite-element method and the analytical method. In this approach, both the propagating and evanescent higher order modes are taken into account, and the restriction in the previous work that the number of assumed waveguides modes be equal to the number of boundary finite-element nodal points is removed. This approach is applicable to the frequency range in which the plate waveguide propagates multimodes. Numerical examples on the scattering by a step discontinuity, a wedge-shaped crack, and a metal strip are given.
\end{abstract}

\section{INTRODUCTION}

S CATTERING of guided waves by discontinuities in an elastic plate is a basic problem in nondestructive evaluation and also has important implications with regard to electrical signal-processing functions, and the scattering of horizontally polarized shear (SH) waves has been investigated extensively [1]-[5].' Recently, the numerical methods based on the finite-element method (FEM) have been developed for the analysis of the scattering of SH waves by arbitrarily shaped discontinuities in an elastic plate. Sabbagh et al. [6] have analyzed the scattering of SH waves by a step discontinuity joining two plates using a combined method of the FEM and circuit theory. In their approach it is assumed that the plate waveguide propagates a single mode only, and all the higher order modes in the waveguide are neglected. Abduljabbar et al. [7] have analyzed the scattering of SH waves by an infinitely thin crack normal to the surface of a plate using a combined method of the FEM and analytical technique. In their approach the higher-order modes are taken into account, but the stresses along the boundary separating the interior finite-element region from the exterior regular region, in which the scattered field is expanded in a superposition of a finite number of normal modes, are assumed to be piecewise uniform. This combined method requires that the number of the nodal points along the interface boundary between the interior and the exterior regions should be equal to that of the normal modes in the waveguide. Koshiba et al. [8] have analyzed the scattering of Lamb waves in an elastic plate using the combined finite-element and analytical technique. In [8] only the fundamental Lamb wave is taken into account, and all the higher order modes in the waveguide are neglected. Edmunds et al. [9] have used the least-squares collocation approach to solve fracture mechanics problems, removing the restriction that the number of terms in a stress function expansion (analogous to the number of waveguide modes in the present problem) be equal to the number of boundary collocation points.

In this paper, an improved formulation of the combined finite-element and analytical technique is described for the analysis of the scattering of SH waves by arbitrarily shaped discontinuities in an elastic plate. In this approach both the propagating and the evanescent higher order modes are taken into account and the stresses along the interface boundary separating the interior finite-element region from the exterior region are discretized by utilizing the shape functions [7], [8], [10]-[14] which are used for a standard procedure in the FEM. Also, the number of the nodal points along the interface boundary and that of the normal modes are independent of each other and the nodal points can be arbitrarily spaced along the interface boundary. This approach is applicable to the frequency range in which the plate waveguide propagates multimodes. To show the validity and usefulness of this formulation, computed results are given for a step discontinuity, a wedgeshaped crack, and a metal strip. Accuracy of the solution is investigated in detail.

\section{BASIC EquATIONS}

We consider the plate waveguide junction as shown in Fig. 1, where the boundaries $\Gamma_{1}$ and $\Gamma_{2}$ connect the discontinuity region to the plate waveguides 1 and 2 , respectively, the boundaries $\Gamma_{3}$ and $\Gamma_{4}$ are the stress-free surface, and the region $\Omega$ with the boundaries $\Gamma_{1}$ to $\Gamma_{4}$ completely encloses the discontinuities.

Assuming that there is no variation in the $z$-direction, we have the following basic equations for $\mathrm{SH}$ waves [1]:

$$
\partial T_{z x} / \partial x+\partial T_{z y} / \partial y-j \omega \rho v_{z}=0
$$

and

$$
\begin{aligned}
& j \omega T_{z x}=\mu \partial v_{z} / \partial x \\
& j \omega T_{z y}=\mu \partial v_{z} / \partial y
\end{aligned}
$$




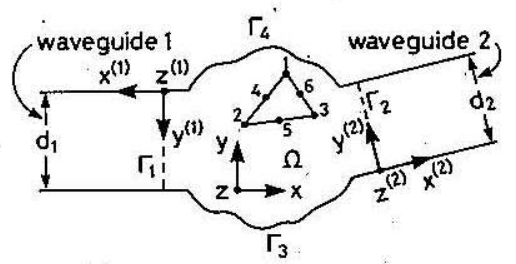

Fig. 1. Discontinuity in elastic plate.

where $v_{z}$ is the $z$ component of the particle velocity, $T_{z x}$ and $T_{z y}$ are the stresses, $\omega$ is the angular frequency, $\rho$ is the mass density, and $\mu$ is the Lamé constant.

\section{Mathematical Formulation}

\section{A. Finite-Element Approach}

Dividing the region $\Omega$ into a number of second-order triangular elements with six nodal points as shown in Fig. 1 , the particle velocity $v_{z}$ within each element may be written as

$$
v_{z}=\{N\}^{T}\left\{v_{z}\right\}_{e}
$$

where $\left\{v_{z}\right\}_{e}$ is the particle velocity vector corresponding to the nodal points within each element, $\{N\}$ is the shape function vector [7], [8], [10]-[14], and $T,\{\cdot\}$, and $\{\cdot\}^{T}$ denote a transpose, a column vector, and a row vector, respectively. The advantage of an arbitrary triangular shape in approximating to any boundary shape has been demonstrated in [10]. Also, the usefulness of the secondorder triangular element has been discussed in [12] and [13].

Using a Galerkin procedure on (1) and integrating by parts, we obtain

$$
\begin{aligned}
& \iint_{e}\left[(\partial\{N\} / \partial x) T_{z x}+(\partial\{N\} / \partial y) T_{z y}\right. \\
& \left.\quad+j \omega \rho_{e}\{N\} v_{z}\right] d x d y-\int_{e}\{N\} T_{z n} d \Gamma=\{0\} .
\end{aligned}
$$

where the first and second integrations on the left side are carried over the element subdomain $\Omega_{e}$ and the contour $\Gamma_{e}$ of $\Omega_{e}$, respectively, $\{0\}$ is a null vector, and

$$
T_{z n}=T_{z x} n_{x}+T_{z y} n_{y}
$$

Here $n_{x}$ and $n_{y}$ are the $x$ and $y$ components of an outward - normal unit vector to $\Gamma_{e}$, respectively.

Noting that $T_{z n}$ is continuous across $\Gamma_{e}$ and $T_{z n}=0$ on $\Gamma_{3}$ and $\Gamma_{4}$, from (2) to (4) the following global matrix equation is derived

$$
[A]\left\{v_{z}\right\}=\sum_{i=1}^{2} \sum_{e}^{\prime} j \omega \int_{e}\{N\}_{i} T_{z x, i}\left(x^{(i)}=0, y^{(i)}\right) d y^{(i)}
$$

where

$$
\begin{aligned}
{[A]=} & \sum_{e} \iint_{e}\left[\mu_{e}(\partial\{N\} / \partial x)\left(\partial\{N\}^{T} / \partial x\right)\right. \\
& +\mu_{e}(\partial\{N\} / \partial y)\left(\partial\{N\}^{T} / \partial y\right) \\
& \left.-\omega^{2} \rho_{e}\{N\}\{N\}^{T}\right] d x d y .
\end{aligned}
$$

Here $\left\{v_{z}\right\}$ is the nodal particle velocity vector, $\Sigma_{e}$ and $\Sigma_{e}^{\prime}$ extend over all different elements and the elements related to $\Gamma_{i}(i=1,2)$, respectively, $T_{z x, i}\left(x^{(i)}=0, y^{(i)}\right)$ is the stress on $\Gamma_{i}$, and $\{N\}_{i}$ is the shape function vector on $\Gamma_{i}$, namely $\{N\}_{i}=\left\{N\left(x^{(i)}=0, y^{(i)}\right)\right\}$.

Using the shape function vector $\{N\}_{i}$, the particle velocity $v_{z, i}$ and the stress $T_{z x, i}$ on $\Gamma_{i}$ may be discretized as follows:

$$
\begin{gathered}
v_{z, i}\left(x^{(i)}=0, y^{(i)}\right)=\{N\}_{i}^{T}\left\{v_{z}\right\}_{i, e} \\
T_{z x, i}\left(x^{(i)}=0, y^{(i)}\right)=\{N\}_{i}^{T}\left\{x_{z x}\right\}_{i, e}
\end{gathered}
$$

where $\left\{v_{z}\right\}_{i, e}$ and $\left\{T_{z x}\right\}_{i, e}$ are the particle velocity vector and the stress vector corresponding to the nodal points within each element related to $\Gamma_{i}$, respectively.

Substituting (8) and (9) into (6), we obtain

$$
[A]\left[\begin{array}{l}
\left\{v_{z}\right\}_{I} \\
\left\{v_{z}\right\}_{B}
\end{array}\right]=\left[\begin{array}{c}
\{0\} \\
{[B]\left\{T_{z x}\right\}_{B}}
\end{array}\right]
$$

where

$$
\begin{aligned}
\left\{v_{z}\right\}_{B} & =\left[\begin{array}{l}
\left\{v_{z}\right\}_{1} \\
\left\{v_{z}\right\}_{2}
\end{array}\right] \\
\left\{T_{z x}\right\}_{B} & =\left[\begin{array}{l}
\left\{T_{z x}\right\}_{1} \\
\left\{T_{z x}\right\}_{2}
\end{array}\right] \\
{[B] } & =\left[\begin{array}{ll}
{[B]_{1}} & {[0]} \\
{[0]} & {[B]_{2}}
\end{array}\right]
\end{aligned}
$$

and

$$
[B]_{i}=j \omega \sum_{e}^{\prime} \int_{e}\{N\}_{i}\{N\}_{i}^{T} d y^{(i)}, \quad i=1,2 .
$$

Here the components of the $\left\{v_{z}\right\}_{I}$ vector are the values of $v_{z}$ at the nodal points in $\Omega$ except $\Gamma_{1}$ and $\Gamma_{2}$, the components of the $\left\{v_{z}\right\}_{i}$ and $\left\{T_{z x}\right\}_{i}$ vectors are the values of $v_{z}$ and $T_{z x}$ at the nodal points on $\Gamma_{i}$, respectively, and [0] is a zero matrix.

\section{B. Analytical Approach}

The internal fields in the waveguide $i(i=1,2)$ in Fig. 1 are represented by the sum of the normal modes as [1], [2], [15]

$$
\begin{aligned}
v_{z, i}\left(x^{(i)}, y^{(i)}\right)= & \sum_{n}\left[a_{i n} \exp \left(-j \beta_{i n} x^{(i)}\right)\right. \\
& \left.+b_{i n} \exp \left(j \beta_{i n} x^{(i)}\right)\right] f_{i n}\left(y^{(i)}\right)
\end{aligned}
$$




$$
\begin{aligned}
-T_{z x, i}\left(x^{(i)}, y^{(i)}\right)= & \sum_{n} Y_{\text {in }}\left[a_{\text {in }} \exp \left(-j \beta_{i n} x^{(i)}\right)\right. \\
& \left.-b_{\text {in }} \exp \left(j \beta_{i n} x^{(i)}\right)\right] g_{\text {in }}\left(y^{(i)}\right)
\end{aligned}
$$

where

$$
\begin{aligned}
\beta_{i n} & =\sqrt{k_{s i}^{2}-\left(n \pi / d_{i}\right)^{2}} \\
Y_{i n} & =\mu_{i} \beta_{i n} / \omega \\
f_{i n}\left(y^{(i)}\right) & =g_{i n}\left(y^{(i)}\right)=\sqrt{l_{n} / d_{i}} \cos \left(n \pi y^{(i)} / d_{i}\right) \\
k_{s i} & =\omega \sqrt{\rho_{i} / \mu_{i}}
\end{aligned}
$$

and

$$
l_{i}= \begin{cases}1, & n=0 \\ 2, & n \neq 0 .\end{cases}
$$

Here $a_{i n}$ and $b_{\text {in }}$ are the amplitudes of the $n$th mode ( $n=$ $0,1,2, \cdots)$ in the waveguide $i$ propagating (or decaying) toward the $+x^{(i)}$ and $-x^{(i)}$ directions, respectively.

Now, assuming that the $m$ th mode $(m=0,1,2, \cdots)$ of unit amplitude is incident from the left of waveguide 1 in Fig. 1, the amplitudes of normal modes may be written as

$$
\begin{aligned}
& b_{1 n}= \begin{cases}1, & n=m \\
0, & n \neq m\end{cases} \\
& b_{2 n}=0 .
\end{aligned}
$$

Considering (15) and (20), $v_{z, i}$ and $T_{z x, i}$ on $\Gamma_{i}$ may be expressed as

$$
\begin{aligned}
v_{z, i}\left(x^{(i)}=0, y^{(i)}\right)= & \delta_{i 1} 2 f_{\mathrm{lm}}\left(y^{(1)}\right) \\
& +\int_{0}^{d_{i}} \sum_{n}\left(1 / Y_{i n}\right) f_{i n}\left(y^{(i)}\right) f_{i n}\left(y_{0}^{(i)}\right) \\
& \cdot\left[-T_{z x, i}\left(x^{(i)}=0, y_{0}^{(i)}\right)\right] d y_{0}^{(i)}
\end{aligned}
$$

$$
\begin{aligned}
-T_{z x, i}\left(x^{(i)}=0, y^{(i)}\right)= & -\delta_{i 1} 2 Y_{1 m} g_{1 m}\left(y^{(1)}\right) \\
& +\int_{0}^{d_{i}} \sum_{n} Y_{i n} g_{i n}\left(y^{(i)}\right) g_{i n}\left(y_{0}^{(i)}\right) \\
& \cdot v_{z, i}\left(x^{(i)}=0, y_{0}^{(i)}\right) d y_{0}^{(i)}
\end{aligned}
$$

where $\delta_{i 1}$ is the Kronecker $\delta$.

Using (8) and (9), (22) and (23) can be discretized as follows:

$$
\begin{aligned}
\left\{v_{z}\right\}_{i} & =\delta_{i 1}\{f\}_{1}-[F]_{i}\left\{T_{z x}\right\}_{i} \\
-\left\{T_{z x}\right\}_{i} & =\delta_{i 1}\{g\}_{1}+[G]_{i}\left\{v_{z}\right\}_{i}
\end{aligned}
$$

where

$$
\begin{aligned}
& \{f\}_{1}=2\left\{f_{m}\right\}_{1} \\
& \{g\}_{1}=-2 Y_{1 m}\left\{g_{m}\right\}_{1}
\end{aligned}
$$

$$
\begin{aligned}
{[F]_{i}=} & \sum_{n}\left(1 / Y_{i n}\right)\left\{f_{n}\right\}_{i} \Sigma_{e}^{\prime} \int_{e} f_{i n}\left(y_{0}^{(i)}\right) \\
& \cdot\left\{N\left(x^{(i)}=0, y_{0}^{(i)}\right)\right\}^{T} d y_{0}^{(i)}
\end{aligned}
$$

and

$$
\begin{aligned}
{[G]_{i}=} & \sum_{n} Y_{i n}\left\{g_{n}\right\}_{i} \sum_{e}^{\prime} \int_{e} g_{i n}\left(y_{0}^{(i)}\right) \\
& \cdot\left\{N\left(x^{(i)}=0, y_{0}^{(i)}\right)\right\}^{T} d y_{0}^{(i)} .
\end{aligned}
$$

Here the components of the $\left\{f_{n}\right\}_{i}$ and $\left\{g_{n}\right\}_{i}$ vectors are the values of $f_{i n}\left(y^{(i)}\right)$ and $g_{i n}\left(y^{(i)}\right)$ at the nodal points on $\Gamma_{i}$, respectively.

\section{Combination of Finite-Element and Analytical \\ Relations}

Using (11) and (12), from (10) and (24) we obtain the following final matrix equation:

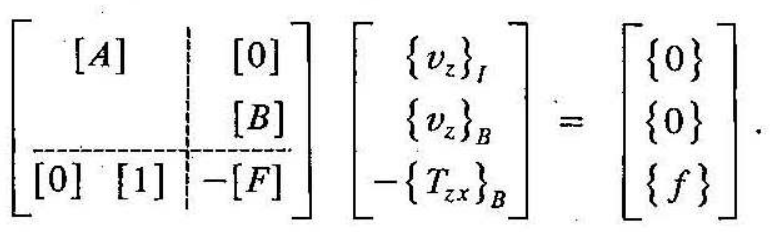

Similarly, from (10) and (25) we obtain the following final matrix equation:

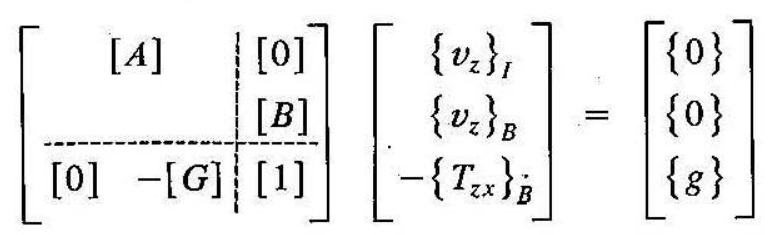

where

$$
\begin{aligned}
& \{f\}=\left[\begin{array}{l}
\{f\}_{1} \\
\{0\}
\end{array}\right] \\
& \{g\}=\left[\begin{array}{l}
\{g\}_{1} \\
\{0\}
\end{array}\right] \\
& {[F]=\left[\begin{array}{ll}
{[F]_{1}} & {[0]} \\
{[0]} & {[F]_{2}}
\end{array}\right]} \\
& {[G]=\left[\begin{array}{ll}
{[G]_{1}} & {[0]} \\
{[0]} & {[G]_{2}}
\end{array}\right]}
\end{aligned}
$$

and [1] is a unit matrix.

The values of $v_{z}$ at the nodal points on $\Gamma_{i}(i=1,2)$, namely $\left\{v_{z}\right\}_{i}$ are computed from (30) or (31), and then by using $(8), v_{z, i}$ on $\Gamma_{i}$ is calculated from these values. The solutions $v_{z, i}$.allow the determination of the reflection coefficient $R_{m n}$ and the transmission coefficient $T_{m n}$ of the $n$th propagating mode for the $m$ th incident mode as fol- 
lows:

$$
\begin{aligned}
R_{m n} \equiv a_{1 n} & =\sqrt{Y_{1 n} / Y_{1 m}}\left[-\delta_{m n}+\int_{0}^{d_{1}} g_{1 n}\left(y^{(1)}\right) v_{z, 1}\left(x^{(1)}\right.\right. \\
& \left.\left.=0, y^{(1)}\right) d y^{(1)}\right] \\
T_{m n} \equiv a_{2 n} & =\sqrt{Y_{2 n} / Y_{1 m}} \int_{0}^{d_{2}} g_{2 n}\left(y^{(2)}\right) v_{z, 2}\left(x^{(2)}\right. \\
& \left.=0, y^{(2)}\right) d y^{(2)}
\end{aligned}
$$

The condition of power conservation may be written as

$$
\sum_{n=0}^{n_{1}-1}\left|R_{m n}\right|^{2}+\sum_{n=0}^{n_{2}-1}\left|T_{m n}\right|^{2} \equiv P_{\text {total }}=1
$$

where $n_{i}$ is the number of the propagating modes in the waveguide $i$.

\section{Computed Results}

First, we consider the symmetric step joining two plates and subdivided one half of the discontinuity region into second-order triangular elements as shown in Fig. 2, where the zeroth mode is incident from the left of the plate with thickness $d_{1}, d_{1} / d_{2}=1.7,96\left(N_{E}\right)$ elements are used, and the number of the nodal points $\left(N_{p}\right)$ is 233 . Table I shows the numbers $n_{1}$ and $n_{2}$ of the propagating modes in the plates with thicknesses $d_{1}$ and $d_{2}$, respectively. Table II shows the real and imaginary parts of the reflection coefficient $R_{00}$ of the zeroth mode at the step, where the computed results are obtained by using (30). Table' III shows the energy error $\left(1-P_{\text {total }}\right)$, where the computed results are obtained by using (30) and (31). $N_{i M}$ $(i=1,2)$ is the number of the normal modes in the waveguide $i$ used in (28) and (29). Here we set $N_{1 M}=N_{2 M} \equiv$ $N_{M}$. In these tables, the results of $N_{E}=384\left(N_{P}=849\right)$ and of $N_{E}=846\left(N_{p}=1849\right)$ are also presented. From Table II it is found that for smaller values of $k_{s} d_{2}$, both values of real and imaginary parts converge with $N_{E}=96$ (see the values for $k_{s} d_{2}=1.0,5.0$, and 10.0 ), but for larger values of $k_{s} d_{2}$, the values of imaginary part do not converge with $N_{E}=96$ (see the values for $k_{s} d_{2}=20.0$ and 30.0). The convergence is obtained in the case of $N_{E}$ $=384$. From Table III it is found that the energy error can be reduced by increasing the number of elements. The larger the value of $N_{M}$ becomes, the larger the energy error also becomes. This is due to the fact that when discretizing the normal modes with larger values of $n$ in (28) and (29), more nodal points on $\Gamma_{i}(i=1,2)$ in Fig. 2 are necessary. In practice, it is sufficient to consider the propagating modes and a few evanescent higher order modes in (28) and (29). The difference between the values calculated from (30) and the values calculated from (31) is relatively small, but, generally, the energy error in the analysis using (31) is larger than that in the analysis using (30). Therefore, we present below only the results ob-

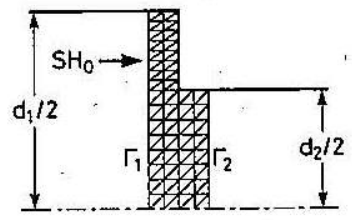

Fig. 2. Typical division of symmetric step.

TABLE I

Numbers of Propagating MOdes in the Plates in Fig. 2

\begin{tabular}{rrr}
\hline \hline$k_{s} d_{2}$ & $n_{1}$ & $n_{2}$ \\
\hline 1.0 & 1 & 1 \\
5.0 & 2 & 1 \\
10.0 & 3 & 2 \\
20.0 & 6 & 4 \\
30.0 & 9 & 5 \\
\hline
\end{tabular}

tained by using (30). It is generally difficult to determine a priori the discretization fineness required to achieve sufficient accuracy. The finite-element method with a suitable mesh refinement strategy [11], [14] may be useful for accurate analysis.

Next, we consider a surface crack in a plate. Results for an infinitely thin crack are given in graphical form by Abduljabbar et al. [7]. Our results agreed perfectly with the results in [7] to the accuracy that can be discerned on the graphs.

Fig. 3(a)-(c) show the magnitude of the reflection coefficient $R_{m n}$ of a wedge-shaped crack as a function of the wedge apex angle $\tan \theta$ for the zeroth $(m=0)$, first ( $m$ $=1)$, and second ( $m=2$ ) incident modes, respectively, where $k_{s} d=13.5$ and the reflection coefficient of the mode of order less than $m$ can be computed from the relation $R_{m n}=R_{n m}$ [7]. The number of the nodal points along the interface boundary corresponding to the boundary $\Gamma_{i}(i=1,2)$ in Fig. 1 is 41 , and 10 normal modes in the waveguide $i$ are considered in (28). The reflection coefficient is considerably influenced by the wedge apex angle. At an appropriate value of $\theta$, the conversion coefficient for the fourth reflected mode becomes larger than the conversion coefficients for the other reflected modes.

Lastly, we consider a metal strip on one surface of a fused quartz plate and assume that the zeroth mode is incident [16]. Fig. 4 shows the magnitude of the reflection coefficient $R_{00}$ of a metal strip as a function of the normalized strip thickness $h / \lambda$, where $\lambda$ is the wavelength of the incident wave, $\lambda=2 \pi / k_{s}, d / \lambda=0.25$, and the normalized strip width $a / \lambda=0.25$. The reflectivity of this $\mathrm{SH}$ wave is considerably large compared with the Rayleigh-wave reflection from a metal strip on a semiinfinite substrate [17]. 
TABLE II

Real and Imaginary Parts of Reflection Coefficient $R_{00}$ of Zeroth Mode

\begin{tabular}{rrrccccc}
\hline \hline$N_{E}$ & & & \multicolumn{3}{c}{ Real Part } \\
$k_{s} d_{2}$ & $N_{M}=15$ & $N_{M}=30$ & $N_{M}=50$ & $N_{M}=15$ & $N_{M}=30$ & $N_{M}=50$ \\
\hline & 1.0 & 0.261 & 0.261 & 0.261 & 0.040 & 0.040 & 0.040 \\
& 5.0 & 0.439 & 0.439 & 0.439 & 0.030 & 0.030 & 0.030 \\
& 10.0 & 0.399 & 0.400 & 0.400 & 0.020 & 0.020 & 0.020 \\
& 20.0 & 0.406 & 0.406 & 0.406 & 0.016 & 0.016 & 0.015 \\
& 30.0 & 0.414 & 0.415 & 0.415 & 0.020 & 0.020 & 0.020 \\
& 1.0 & 0.261 & 0.261 & 0.261 & 0.040 & 0.040 & 0.040 \\
& 5.0 & 0.440 & 0.440 & 0.440 & 0.030 & 0.030 & 0.030 \\
& 10.0 & 0.400 & 0.400 & 0.400 & 0.020 & 0.020 & 0.020 \\
& 20.0 & 0.406 & 0.406 & 0.406 & 0.012 & 0.012 & 0.012 \\
& 30.0 & 0.414 & 0.414 & 0.414 & 0.010 & 0.010 & 0.010 \\
& 20.0 & 0.407 & 0.407 & 0.407 & 0.012 & 0.012 & 0.012 \\
& 30.0 & 0.414 & 0.414 & 0.414 & 0.009 & 0.009 & 0.009 \\
\hline
\end{tabular}

TABLE III

ENERGY ERRoR IN THE ANALYSIS OF STEP DisCONTINUITY IN Fig. 2

\begin{tabular}{|c|c|c|c|c|c|c|c|}
\hline$N_{E}$ & $k_{s} d_{2}$ & $N_{M}=15$ & $\begin{array}{l}\text { Equation (30) } \\
N_{M}=30\end{array}$ & $N_{M}=50$ & $N_{M}=15$ & $\begin{array}{l}\text { Equation (31) } \\
\qquad N_{M}=30\end{array}$ & $N_{M}=50$ \\
\hline 96 & $\begin{array}{r}1.0 \\
5.0 \\
10.0 \\
20.0 \\
30.0\end{array}$ & $\begin{array}{l}3.2 \times 10^{-8} \\
1.2 \times 10^{-6} \\
7.4 \times 10^{-7} \\
1.6 \times 10^{-5} \\
6.9 \times 10^{-5}\end{array}$ & $\begin{array}{l}6.0 \times 10^{-7} \\
1.0 \times 10^{-5} \\
7.0 \times 10^{-5} \\
5.1 \times 10^{-4} \\
2.2 \times 10^{-3}\end{array}$ & $\begin{array}{r}3.1 \times 10^{-7} \\
-4.3 \times 10^{-6} \\
9.8 \times 10^{-5} \\
3.0 \times 10^{-4} \\
2.2 \times 10^{-3}\end{array}$ & $\begin{array}{r}-3.6 \times 10^{-6} \\
-2.3 \times 10^{-6} \\
-1.8 \times 10^{-6} \\
4.2 \times 10^{-5} \\
-4.3 \times 10^{-5}\end{array}$ & $\begin{array}{r}-3.4 \times 10^{-4} \\
-3.0 \times 10^{-4} \\
9.8 \times 10^{-5} \\
3.0 \times 10^{-4} \\
-8.8 \times 10^{-5}\end{array}$ & $\begin{array}{l}-4.3 \times 10^{-5} \\
-1.1 \times 10^{-4} \\
-5.8 \times 10^{-4} \\
-1.1 \times 10^{-3} \\
-2.2 \times 10^{-3}\end{array}$ \\
\hline 384 & $\begin{array}{r}1.0 \\
5.0 \\
10.0 \\
20.0 \\
30.0\end{array}$ & $\begin{array}{l}1.9 \times 10^{-9} \\
7.2 \times 10^{-9} \\
3.9 \times 10^{-8} \\
8.8 \times 10^{-7} \\
4.1 \times 10^{-6}\end{array}$ & $\begin{array}{l}1.9 \times 10^{-9} \\
7.3 \times 10^{-8} \\
4.2 \times 10^{-8} \\
8.1 \times 10^{-7} \\
4.5 \times 10^{-6}\end{array}$ & $\begin{array}{l}6.2 \times 10^{-9} \\
6.0 \times 10^{-7} \\
2.0 \times 10^{-6} \\
2.3 \times 10^{-5} \\
3.2 \times 10^{-6}\end{array}$ & $\begin{array}{l}-2.0 \times 10^{-7} \\
-1.2 \times 10^{-7} \\
-7.5 \times 10^{-8} \\
-5.3 \times 10^{-7} \\
-4.8 \times 10^{-6}\end{array}$ & $\begin{array}{r}-2.2 \times 10^{-7} \\
-1.3 \times 10^{-7} \\
-1.1 \times 10^{-7} \\
1.2 \times 10^{-5} \\
8.2 \times 10^{-5}\end{array}$ & $\begin{array}{r}-3.4 \times 10^{-6} \\
1.9 \times 10^{-5} \\
1.3 \times 10^{-4} \\
4.6 \times 10^{-4} \\
1.0 \times 10^{-3}\end{array}$ \\
\hline 864 & $\begin{array}{l}20.0 \\
30.0\end{array}$ & $\begin{array}{l}1.7 \times 10^{-7} \\
7.3 \times 10^{-7}\end{array}$ & $\begin{array}{l}1.7 \times 10^{-7} \\
7.3 \times 10^{-7}\end{array}$ & $\begin{array}{l}4.9 \times 10^{-6} \\
1.7 \times 10^{-5}\end{array}$ & $\begin{array}{l}-1.1 \times 10^{-7} \\
-9.9 \times 10^{-7}\end{array}$ & $\begin{array}{l}-1.1 \times 10^{-7} \\
-9.8 \times 10^{-7}\end{array}$ & $\begin{array}{l}2.0 \times 10^{-4} \\
4.8 \times 10^{-4}\end{array}$ \\
\hline
\end{tabular}

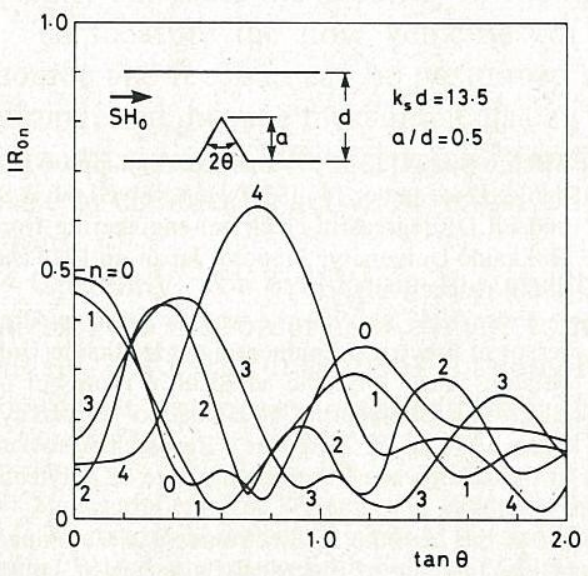

(a)

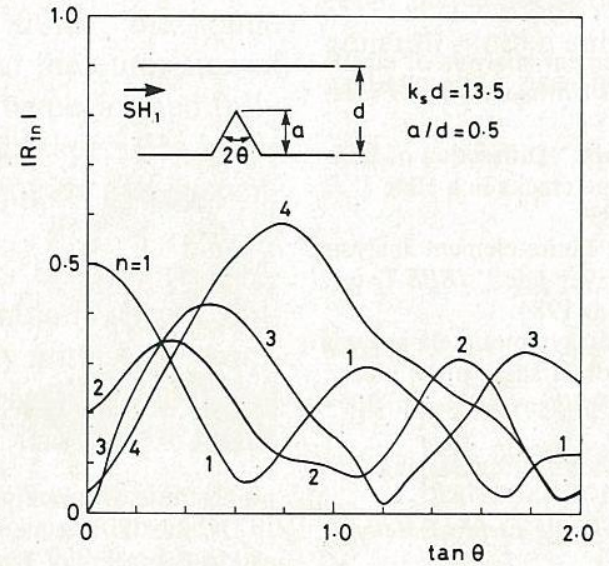

(b)

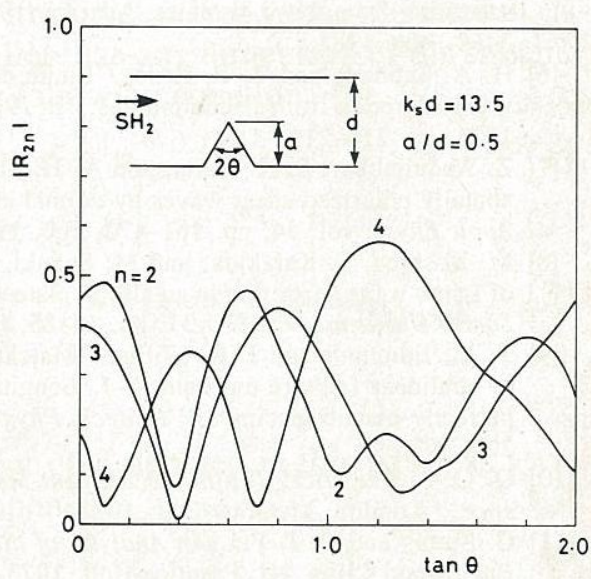

(c)

Fig. 3. Reflection coefficient of wedge-shaped crack for (a) zeroth, (b) first, and (c) second incident modes. 


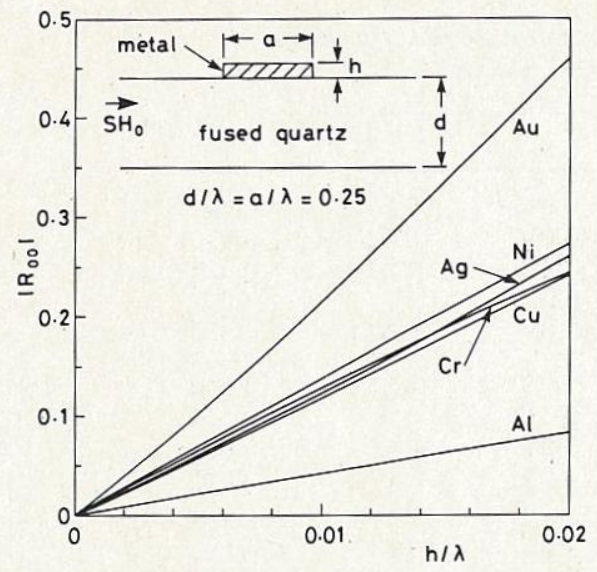

Fig. 4. Reflection coefficient of metal strip for zeroth incident mode.

\section{CONCLUSION}

A method of analysis, based on the finite-element approach and the analytical approach, was developed for the solution of scattering of SH waves in an elastic plate. This method is useful for inhomogeneous discontinuities of arbitrary shape. Numerical examples are presented for a step discontinuity, a wedge-shaped crack, and a metal strip.

\section{ACKNOWLEDGMENT}

The authors wish to thank S. Karakida for his contribution in the earlier part of this work.

\section{REFERENCES}

[1] B. A. Auld, Acoustic Fields and Waves in Solids, Vol. II. New York: Wiley-Interscience, 1973.

[2] S. Sato and T. Makimoto, "Variational expression for scattering coefficients at piezoelectric waveguide step junction,"' Trans. Inst. Electron. Commun. Eng. Japan, vol. 58-B, pp. 231-238, May 1975 (in Japanese).

[3] S. K. Datta, A. H. Shah, and C. M. Fortunko, "Diffraction of medium and long wavelength horizontally polarized shear waves by edge crack," J. Appl. Phys., vol. 53, pp. 2895-2903, Apr. 1982.

[4] C. M. Fortunko, R. B. King, and M. Tan, "Nondestructive evaluation of planar defects in plates using low-frequency shear horizontal waves," J.Appl. Phys., vol. 53, pp. 3450-3458, May 1982.

[5] R. B. King and C. M. Fortunko, "Extended variational solution for scattering from flaws in plates," J. Appl. Phys., vol. 53, pp. 34593460, May 1982.

[6] H. A. Sabbagh and T. F. Krile, "Finite element analysis of elastic wave scattering from discontinuities," in 1973 Ultrason. Symp. Proc., 1973, pp. 216-219.

[7] Z. Abduljabbar, S. K. Datta, and A. H. Shah, "Diffraction of horizontally polarized shear waves by normal edge cracks in a plate," $J$. Appl. Phys., vol. 54, pp. 461-472, Feb. 1983

[8] M. Koshiba, S. Karakida, and M. Suzuki, "Finite-element analysis of Lamb wave scattering in an elastic plate waveguide," IEEE Trans. Sonics Ultrason., vol. SU-31, pp. 18-25, Jan. 1984.

[9] T. M. Edmunds and J. R. Willis, "Matched asymptotic expansions in nonlinear fracture mechanics-I. Longitudinal shear of an elastic perfectly-plastic specimen,"'J. Mech. Phys. Solids, vol. 24, pp. 205223, 1976.

[10] O. C. Zienkiewicz, The Finite Element Method in Engineering Science. London: McGraw-Hill, 1971.

[11] G. Strang and G. J. Fix, An Analysis of the Finite Element Method. Englewood Cliffs, NJ: Prentice-Hall, 1973.

[12] P. E. Lagasse, "Higher-order finite-element analysis of topographic guides supporting elastic surface waves," J. Acoust. Soc. Am., vol. 53, pp. 1116-1122, Apr. 1973.
[13] P. E. Lagasse, "Finite element analysis of piezoelectric elastic waveguides," IEEE Trans. Sonics Ultrason., vol. SU-20, pp. 354-359, Oct. 1973.

[14] A. J. Jennings and G. K. Cambrell, "Refined finite-element analysis of a clad fiber acoustic waveguide," IEEE Trans. Sonics Ultrason., vol. SU-29, pp. 239-248, Sept. 1982.

[15] S. Sato and T. Makimoto, "Stationary expression for scattering coefficients at uniform piezoelectric waveguide junction," Proc. IEEE, vol. 61, pp. 1648-1650, Nov. 1973.

[16] E. K. Sittig and G. A. Coquin, "Filters and dispersive delay lines using repetitively mismatched ultrasonic transmission lines," IEEE Trans. Sonics Ultrason., vol. SU-15, pp. 111-119, Apr. 1968.

[17] H. Matthews, Ed., Surface Wave Filters, New York: Wiley-Interscience, 1977

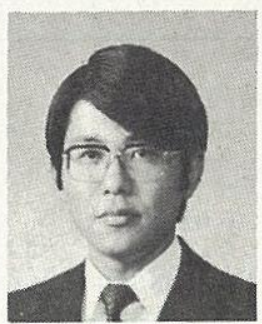

Masanori Koshiba (SM'84) was born in Sapporo, Japan, on November 23, 1948. He received the B.S., M.S., and Ph.D. degrees in electronic engineering from Hokkaido University, Sapporo, Japan, in 1971, 1973, and 1976, respectively.

In 1976, he joined the Department of Electronic Engineering, Kitami Institute of Technology, Kitami, Japan. Since 1979, he has been an Assistant Professor of Electronic Engineering at Hokkaido University. He has been engaged in research on surface acoustic waves, dielectric optical waveguides, and applications of finite-element and boundary-element methods to field problems.

Dr. Koshiba is a member of the Institute of Electronics and Communication Engineers of Japan, the Institute of Television Engineers of Japan, the Institute of Electrical Engineers of Japan, the Japan Society for Simulation Technology, and Japan Society for Computation Methods in Engineering.

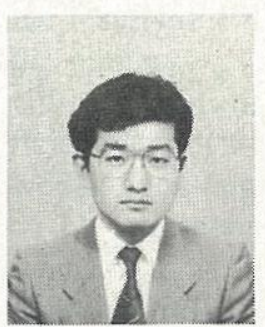

Koji Hasegawa was born in Fukagawa, Japan, on October 2, 1961. He received the B.S. degree in electronic engineering from Hokkaido University, Sapporo, Japan, in 1984.

He is presently studying toward the M.S. degree in electronic engineering, Hokkaido University, Sapporo, Japan.

Mr. Hasegawa is a member of the Institute of Electronics and Communication Engineers of Japan.

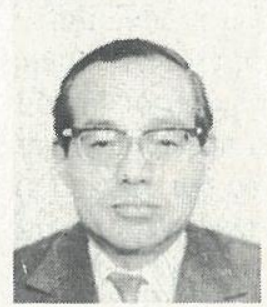

Michio Suzuki (SM'57) was born in Sapporo, Japan, on November 14, 1923. He received the B.S and $\mathrm{Ph} . \mathrm{D}$. degrees in electrical engineering from Hokkaido University, Sapporo, Japan, in 1946 and 1960, respectively.

From 1948 to 1962, he was an Assistant Professor of Electrical Engineering at Hokkaido University. Since 1962, he has been a Professor of Electronic Engineering at Hokkaido University. From 1956 to 1957, he was a Research Associate at the Microwave Research Institute of Polytechnic Institute of Brooklyn, Brooklyn, NY.

Dr. Suzuki is a member of the Institute of Electronics and Communication Engineers of Japan, the Institute of Electrical Engineers of Japan, the Institute of Television Engineers of Japan, the Japan Society of Information and Communication Research, and the Japan Society for Simulation Technology. 